\title{
Population-based reversible jump Markov chain Monte Carlo
}

\author{
AJAY JASRA ${ }^{\star}$, DAVID A. STEPHENS* \& CHRISTOPHER C. HOLMES ${ }^{\dagger}$ \\ ${ }^{\star}$ Department of Mathematics, Imperial College London, SW7 2AZ, London, UK \\ *Department of Mathematics and Statistics, McGill University, H3A 2K6, Montreal, CA \\ ${ }^{\dagger}$ Department of Statistics, University of Oxford, OX1 3TG, Oxford, UK
}

\begin{abstract}
In this paper we present an extension of population-based Markov chain Monte Carlo (MCMC) to the trans-dimensional case. One of the main challenges in MCMC-based inference is that of simulating from high and trans-dimensional target measures. In such cases, MCMC methods may not adequately traverse the support of the target; the simulation results will be unreliable. We develop population methods to deal with such problems, and give a result proving the uniform ergodicity of these population algorithms, under mild assumptions. This result is used to demonstrate the superiority, in terms of convergence rate, of a population transition kernel over a reversible jump sampler for a Bayesian variable selection problem. We also give an example of a population algorithm for a Bayesian multivariate mixture model with an unknown number of components. This is applied to gene expression data of 1000 data points in six dimensions and it is demonstrated that our algorithm out performs some competing Markov chain samplers.

Some key words: Population Monte Carlo, Uniform ergodicity, Bayesian variable selection, Mixture models, Gene expression data
\end{abstract}

\section{Introduction}

The Metropolis-Hastings algorithm (Metropolis et al. 1953; Hastings, 1970) and its adaptation to the transdimensional case (Green, 1995) has provided a method to simulate from complex probability measures in high dimensions. This has facilitated the application of (particularly Bayesian) complicated statistical models which could not otherwise be fitted.

We consider the problem of simulating from a probability measure $\pi(x) \lambda(d x)$ defined on measurable space $(E, \mathcal{E})$ (where $\lambda$ is a $\sigma$-finite measure on $\mathcal{E}$ ), with $\pi$ known pointwise, at least up to a normalizing constant. This is achieved (in the context of MCMC) by simulating an ergodic Markov chain $\left\{X_{n}\right\}_{n \geq 0}$, with kernel $K: E \times \mathcal{E} \rightarrow[0,1]$, of stationary distribution $\pi$. The Markov chain can be used, for example, to estimate expectations of $\pi$-integrable functions. In this article we focus on reversible jump Markov 
chain Monte Carlo (RJMCMC), where the state space is a union of subspaces of differing dimension, that is, where $E=\bigcup_{k \in \mathcal{K}}\left(\{k\} \times E^{k}\right), \mathcal{K} \subseteq \mathbb{N}, E^{k} \subseteq \mathbb{R}^{k}$.

In statistical terms, $\pi$ will often be a Bayesian posterior distribution which is normally known pointwise, up to a normalizing constant. For example, applications of RJMCMC include classification and regression (Denison et al., 2002) and mixture modelling (Richardson and Green, 1997), with particular emphasis on model determination. However, in many examples, the naive (vanilla) RJMCMC sampler can fail to move around the support of the target in a feasible computation time; see Brooks et al. (2003) for examples.

To deal with these problems, several MCMC approaches have been suggested, including auxiliary variable methods (Brooks et al., 2003) and tempered transitions (Jennison et al., 2003): Green (2003b) provides a recent review. One approach used for difficult sampling problems in fixed dimensional spaces, that have not been widely used in the variable dimension case, relies on population-based MCMC methods (Liang \& Wong, 2001; Liu, 2001). It is straightforward, conceptually, to extend this approach to the variable dimension case. Such an extension is the focus of this paper; we study the potential theoretical and practical advantages of population approaches over standard MCMC methods. We remark that methods other than MCMC may be used for difficult simulation problems, such as sequential Monte Carlo (e.g. Del Moral et al., (2006)), but such methods are not the focus of this paper.

\subsection{Population-based Markov chain Monte Carlo}

Population-based MCMC operates by embedding the target into a sequence of related probability measures $\left\{\pi_{i}\right\}_{i \in \mathbb{T}_{N}}, \mathbb{T}_{N}:=\{1, \ldots, N\}$, and simulating the $N$ parallel chains (the population), as in parallel tempering (Geyer, 1991; Hukushima \& Nemoto, 1996). In addition, the chains are allowed to interact via various crossover moves; a summary is given in Section 3 - see Liu (2001) for an extensive review.

The main advantage of population-based simulation over other methods is the fact that the population simultaneously represents many properties of the target distribution. This is particularly useful in transdimensional simulation, where it can be difficult to construct efficient dimension-changing proposals. Green (2003b) notes that some MCMC methods retain information about which states have been visited, for

example, the product space approach (Carlin \& Chib, 1995; Godsill, 2001), whilst standard reversible jump MCMC does not retain this information. There are advantages to both approaches. The first approach can provide an improved mode-jumping property; that is, the ability to jump a large number of dimensions that would take a substantial time under the standard approach. The second approach has greater capacity to discover new states that are consistent with the target. It is clear that an algorithm which can combine these properties is likely to provide an improvement over both methods; the objective of this paper is to construct such an algorithm, and to investigate its theoretical properties. 


\subsection{Contribution and Structure of Paper}

Theoretical aspects of population-based MCMC have been rarely considered (see, however, Madras and Zheng (2003)), and therefore the improvements, if any, offered by population methods over standard MCMC have not been fully established. In this article we present a result which ensures (under fairly mild conditions, including that the density $\pi$ is upper bounded) the uniform ergodicity of a population transition kernel, and allows the construction of population algorithms which are preferable, in theory, to their single chain counterparts. The result can help to illuminate, for small $N$, why population algorithms can work well in practice.

We also demonstrate that a particular population-based kernel - the exchange kernel (see Sections 3.4 and 5.1) which is fundamental to swapping information in population MCMC - can improve convergence properties (over parallel MCMC, in which $N$ independent identical Markov chains are run) under strong assumptions; this has not, to our knowledge, been established previously. Our results are substantiated with an example in Bayesian variable selection, and illustrate the use of population MCMC methods in a mixture problem.

Population methods naturally accommodate multiple MCMC strategies which improve the ability of the sampler to mix across the state space. In our main example we show how to combine the methods of parallel chains (Geyer, 1991), tempering (e.g. Geyer \& Thompson, 1995), snooker algorithms (Gilks, et. al., 1994), constrained sampling (e.g. the unpublished technical report of Atachdé \& Liu (2004)) and delayed rejection (Green \& Mira, 2001). We believe that although such methods may not perform adequately (individually) together they can provide a superior MCMC sampler.

This paper is organised as follows. In Section 2 we provide an illustrative example related to the clustering of gene expression data via Bayesian multivariate mixture models. In Section 3 we introduce population-based reversible jump. In Section 4 we give some theoretical results that can indicate why population methods can perform well. In Section 5 we present population MCMC moves for the mixture model example. In Section 6 we provide a comparison of vanilla, simulated tempering (Geyer \& Thompson, 1995) and population samplers for the mixture model example. In Section 7 we conclude with a discussion, detailing extensions to our approach.

\subsection{Notation}

The notation and mathematical objects that are adopted in the paper are summarized here.

A measurable space is denoted $(E, \mathcal{E})$ : throughout this article $\mathcal{E}$ is a countably generated $\sigma-$ algebra. The product $\sigma$-algebra is written $\mathcal{E}^{N}:=\mathcal{E} \otimes \cdots \otimes \mathcal{E}$ (product $N$-times). We use $\delta_{x}\left(d x^{\prime}\right)$ to represent

Dirac measure 
For two probability measures, $\lambda_{1}$ and $\lambda_{2}$, on $\mathcal{E}$, the total variation distance is written $\left\|\lambda_{1}-\lambda_{2}\right\|_{T V}:=$ $\sup _{A \in \mathcal{E}}\left|\lambda_{1}(A)-\lambda_{2}(A)\right|$. The Dobrushin coefficient (Dobrushin, 1956) of a Markov kernel $K$ is denoted $\beta(K):=\sup _{(x, y) \in E^{2}}\|K(x, \cdot)-K(y \cdot)\|_{T V}$. The composition of two Markov kernels, $K$ and $P$ is written $K \circ P(x, d y):=\int_{E} K(x, d u) P(u, d y)$, except if $K=P$ where $K^{2}$ is used. Particular Markov kernels of interest are the product and mixture kernels that combine component kernels $K_{i}$ in a multiplicative and additive fashion, which will be denoted

$$
\prod_{i} K_{i} \quad \sum_{i} \tau_{i} K_{i}
$$

for mixture weights $\tau_{i}$. Given a probability measure $\lambda$ and Markov kernel $K$, the standard notation $\lambda K(d y):=\int_{E} \lambda(d x) K(x, d y)$ is adopted. For a bounded measurable function $f$, the oscillations are written $\operatorname{osc}(f)=\sup _{(x, y) \in E^{2}}|f(x)-f(y)|$.

Finally, we use the vector notation $x_{1: N}:=\left(x_{1}, \ldots, x_{N}\right)$ and denote a vector with its $i^{\text {th }}$ element missing as $x_{-i}$ and with only its $i^{\text {th }}$ and $j^{\text {th }}$ elements as $x_{i, j}$. Also, $\mathbb{T}_{l: p}:=\{l, \ldots, p\}$ for any $l \leq p,(l, p) \in \mathbb{Z}^{2}$.

\section{An Illustrative Example: Finite Mixture Modelling}

Mixture models are typically used to model heterogeneous data, or as a simple means of density estimation; see McLachlan \& Peel (2000) for an overview. Bayesian analysis using mixtures with an unknown number of components has only fairly recently been implemented (see Richardson \& Green (1997) and in the multivariate context Stephens (2000) and Dellaportas \& Papageorgiou (2006)).

In this section, we describe the finite mixture model adopted and a motivating example, in which standard MCMC methods do not perform adequately.

\subsection{Model}

Let $y_{1: n}$ denote observed data that lie on support $y_{i} \in Y \subseteq \mathbb{R}^{r}, i=1, \ldots, n$. We assume that the $y_{i}$ are i.i.d with density:

$$
p\left(y_{i} \mid \eta_{1: k}, w_{1: k}\right)=\sum_{j=1}^{k} w_{j} f\left(y_{i} ; \eta_{j}\right)
$$

where $\eta_{1: k}$ are component specific parameters, the weights $w_{1: k}$ are such that $\sum_{j=1}^{k} w_{j}=1, w_{j} \geq 0 \forall j$, $p$ denotes an arbitrary probability density function and $f$ is the component density. For our model, we restrict ourselves to the case of multivariate $t, \mathcal{T}_{r}(\mu, \Lambda, s)$, where $(\mu, \Lambda)$ are the location and covariance parameters and $s$ is the degrees of freedom.

In specifying the prior distributions, we follow Stephens (2000); the component mean vectors are taken to be independent $\mathcal{N}_{r}\left(\xi, \kappa^{-1}\right)$ (multivariate normal distribution), and the $\Lambda_{j}$ are independently $\mathcal{I} \mathcal{W}_{r}(2 \alpha, 2 \Psi)$, 
where $\operatorname{IW}_{r}(\cdot, \cdot)$ is the inverse Wishart distribution. The following hierarchical structure is adopted:

$$
\begin{aligned}
\Psi & \sim \mathcal{W}_{r}\left(2 g,(2 h)^{-1}\right) \quad \text { where } \mathcal{W}_{r}(\cdot, \cdot) \text { is the Wishart distribution } \\
w_{1: k-1} \mid k & \sim \mathcal{D}(\delta) \quad \text { where } \mathcal{D}(\cdot) \text { is the symmetric Dirichlet distribution } \\
k & \sim \mathcal{U}_{\left\{1, \ldots, k_{\max }\right\}} \quad \text { where } \mathcal{U}_{S} \text { is the discrete uniform distribution on countable set } S .
\end{aligned}
$$

When using the multivariate $t$-distribution, the degrees of freedom are assumed known. Thus, our prior is:

$$
p\left(w_{1: k-1}, \mu_{1: k}, \Lambda_{1: k}, \Psi, k\right)=\left[\prod_{j=1}^{k} p\left(\mu_{j}\right) p\left(\Lambda_{j} \mid \Psi\right)\right] p(\Psi) p\left(w_{1: k-1} \mid k\right) p(k) .
$$

\subsection{Data Processing and Prior Distributions}

For our example we consider the problem of clustering gene expression data ( e.g. Heard et al. (2006)). The data consist of the relative level of gene expression - a measure of genetic activity - for $n=4221$ genes of the parasite Plasmodium measured at $r=46$ time points across a 48 hour portion of the parasite life cycle. The data are discussed in detail in Bozdech et al. (2003). Finding meaningful subgroupings of the data is an important task for biological investigators.

Even with modern computing power, applying a fully Bayesian analysis to such data is not practical. Therefore, the data is preprocessed the to reduce the $n \times r$-dimensional data to $l \times q$ dimensions. We achieve this by adopting a $\mathrm{K}$-means partitioning approach to reduce $n$ to $l$, and then principal components to reduce $r$ to $q$. We selected $l=1000$ and $q=6$.

Prior hyperparameters are set in a similar way to Stephens (2000); we set $\xi$ to be the midpoint of the observed data in its corresponding dimension, and $\kappa$ is taken to be $\operatorname{diag}\left(1 / R_{1}^{2}, \ldots, 1 / R_{q}^{2}\right)$ where $R_{d}$ is the range of the data in dimension $d \in \mathbb{T}_{q}$. Additionally $g=q / 2, \delta=1$ and $\alpha=\alpha^{\prime}+(q+1) / 2$, where $\alpha^{\prime}=3$.

Finally $h$ is $\operatorname{diag}\left(100 q /\left(2 \alpha R_{1}^{2}\right), \ldots, 100 q /\left(2 \alpha R_{q}^{2}\right)\right)$. For illustration a $t$-distribution with four degrees of freedom is used as the component density in our mixture model and set $k_{\max }=20$.

\subsection{Performance of Vanilla Sampler}

A vanilla reversible jump sampler (outlined in Appendix 1) was implemented for the data above. We ran the reversible jump algorithm for 250000 sweeps from two different starting points, which had different initial $k$. The $\mathrm{C}$ program was run on a Pentium 4, $3 \mathrm{Ghz}$ machine and took approximately three hours. We observed extremely poor mixing, with all variable dimension acceptance rates below $1 \%$. It can be seen that there appears to be support for $k \in \mathbb{T}_{3: 5}$ components, but also for $k \in \mathbb{T}_{8: 11}$. The main problem is that the vanilla sampler cannot jump between these two modes.

The poor performance of the vanilla algorithm can be partly attributed to the difference in dimensionality between different mixture models. To jump from a $k$ to a $k+1$ component mixture model, we 
need to draw $1+q+q(q+1) / 2=28$ random variables, so we will need proposals/jump functions that are more tailored than those used in the vanilla sampler. In addition, if between-model jumps are made infrequently, a within-model chain spends a long time in a high density region of the state space specific to that model that is consistent with the data. This within-model adaptation renders a jump between models even less likely, as high density regions in different models do not necessarily correspond to each other in a straightforward fashion. This heuristic argument applies to local moves, and whilst more global moves might be constructed (as noted by Green (2003a), they are more likely to produce better mixing than the local moves attempted here), dimension matching dictates that this will be difficult to achieve efficiently.

\subsection{Alternative Algorithms}

Possible MCMC methods that might be used to deal with the problems encountered here may be the auxiliary variable method of Brooks et al. (2003), but this will require a reasonable movement of the chain in the first place. Constructing proposal distributions by creating an approximation of the target in each dimension using fixed dimensional MCMC (Hastie, 2005 (University of Bristol PhD thesis)) is complicated by the label-switching problem (see Jasra et al. (2005) for a discussion). Delayed rejection (Green \& Mira, 2001) and tempered transitions (Jennison et al., 2003) often do not provide a general solution to the problems highlighted by this example. For the former method, insufficient information is learnt at the first stage rejection to provide a significantly improved second stage proposal, whereas for the latter, it often takes a large number of intermediate simulations (e.g. 100) to provide a reasonable proposal, but even so, the performance gain is not always substantial.

\section{Population-Based Reversible Jump}

We now consider population-based reversible jump algorithms. First we give details of the population MCMC method, and then study the theoretical properties of the algorithm, in particular its uniform ergodicity.

\subsection{The Population MCMC Method}

Consider a sequence of probability measures $\left\{\pi_{i}\right\}_{i \in \mathbb{T}_{N}}$, each assumed to admit a density (also denoted $\pi_{i}$ ) with respect to $\lambda$ on $(E, \mathcal{E})$. Denote the support of the $i^{t h}$ density $E_{i}=\left\{\left(x_{k}, k\right) \in E: \pi_{i}\left(x_{k}, k\right)>0\right\}$, $i \in \mathbb{T}_{N}$. Define probability measure $\pi^{*}\left(d\left(x_{1: k_{1}}, k_{1}, \ldots, x_{1: k_{N}}, k_{N}\right)\right)$ on $\left(E^{N}, \mathcal{E}^{N}\right)$ as:

$$
\pi^{*}\left(d\left(x_{1: k_{1}}, k_{1}, \ldots, x_{1: k_{N}}, k_{N}\right)\right)=\left[\prod_{i=1}^{N} \pi_{i}\left(x_{1: k_{i}}, k_{i}\right) \lambda\left(d\left(x_{1: k_{i}}, k_{i}\right)\right)\right] .
$$


Our objective is to now construct an ergodic Markov kernel $\widetilde{K}: E^{N} \times \mathcal{E}^{N} \rightarrow[0,1]$ with stationary distribution $\pi^{*}$.

\subsection{The Structure of the Population}

The population approach proceeds as follows; we generate $N$ parallel (variable dimension) chains in order to explore the target correctly. For the remainder of the paper $\pi_{1} \equiv \pi$ and the sequence of densities are taken $\pi_{i} \propto \pi^{\zeta_{i}}, 1=\zeta_{1}>\cdots>\zeta_{N}>0$, where $\left\{\zeta_{i}\right\}_{i \in \mathbb{T}_{N}}$ are inverse temperature parameters; the collection $\left\{\zeta_{i}\right\}$ is referred to as the temperature ladder. This is the approach in Liang \& Wong (2001), but other settings include taking $\zeta_{i}=1$ for each $i$; see Del Moral et al. (2006) for further discussion. We seek to use the extra information contained in $N$ chains at different temperatures to allow large moves in dimension of the chain of interest as well as allowing improved performance in more local moves (within and between dimensions).

One of the main problems of parallel tempering (Geyer, 1991; Hukushima \& Nemoto, 1996) is that only minimal interactions between the chains are allowed. Our approach differs as we will allow $\widetilde{K}$ to include moves which use the entire population, other than merely the exchange move (see Section 3.4). Thus we seek a more population-based approach to justify the increased cost in computation.

We now investigate some theoretical aspects of population algorithms. As our results are not confined to the trans-dimensional case, we drop the $k$ from the notation. That is, $E$ is a general state space with associated countably generated $\sigma$-algebra $\mathcal{E}$.

\subsection{Some Theory for Markov chains}

We will concentrate on the concept of uniform ergodicity (see Roberts \& Rosenthal (2004) and the references therein). Our objective is to utilize the following property of uniformly ergodic Markov kernels $K$; the total variation distance between the $n$-step kernel and its stationary measure $\pi$, $\left(\left\|K^{n}(x, \cdot)-\pi(\cdot)\right\|_{T V}\right)$, is bounded above by:

$$
R=(1-\epsilon)^{\left\lfloor\frac{n}{n_{0}}\right\rfloor}
$$

where $n_{0}$ and $\epsilon$ are parameters in the following minorization condition (for $C=E$ ): $\exists \epsilon>0$ such that for $\nu$ some non-trivial probability measure and integer $n_{0}>0$ and $\forall x \in C, A \in \mathcal{E}$ :

$$
K^{n_{0}}(x, A) \geq \epsilon \nu(A)
$$

If $C \in \mathcal{E}$ is such that (3.1) is satisfied, then we term it $\left(n_{0}, \epsilon, \nu\right)$-small.

It follows that, to compare convergence speed (note that spectral gap techniques - see, for example, Diaconis \& Saloff Coste (1993) - can also be considered; see Section 4.3) of two uniformly ergodic Markov 
kernels, we can compute the pair $\left(n_{0}, \epsilon\right)$; if one of the kernels has a substantially larger $\epsilon$ (and smaller $\left.n_{0}\right)$, then we might expect it to converge more quickly to the target of interest.

\subsection{Main Result}

We now demonstrate that population MCMC approaches can be defined, using specially constructed component kernels, so that they are uniformly ergodic. For the following Theorem we denote a mutation (Markov transition) kernel as $K_{M}$ and an exchange kernel $K_{E}$. A mutation move is a Metropolis-Hastings $(\mathrm{MH})$ kernel which attempts to change a single member of the population, dependent only on the current state of the chain for that member. An exchange move is a Metropolis-Hastings kernel which proposes to swap the current states of two different members of the population, mathematically it is defined as, $K_{E}^{(i l)}: E^{2} \times \mathcal{E}^{2} \rightarrow[0,1]:$

$$
\begin{aligned}
K_{E}^{(i l)}\left(x_{i, l}, d x_{i, l}^{\prime}\right)= & \min \left\{1, \frac{\pi_{i}\left(x_{l}\right) \pi_{l}\left(x_{i}\right)}{\pi_{i}\left(x_{i}\right) \pi_{l}\left(x_{l}\right)}\right\} \delta_{x_{l}}\left(d x_{i}^{\prime}\right) \delta_{x_{i}}\left(d x_{l}^{\prime}\right)+ \\
& {\left[1-\min \left\{1, \frac{\pi_{i}\left(x_{l}\right) \pi_{l}\left(x_{i}\right)}{\pi_{i}\left(x_{i}\right) \pi_{l}\left(x_{l}\right)}\right\}\right] \delta_{x_{i}}\left(d x_{i}^{\prime}\right) \delta_{x_{l}}\left(d x_{l}^{\prime}\right) . }
\end{aligned}
$$

For simplicity, we assume that $E_{i}=E \forall i \in \mathbb{T}_{N}$. We now give our main theoretical result;

Theorem 1. Let $\widetilde{K}$ be a Markov kernel defined on a measurable space $\left(E^{N}, \mathcal{E}^{N}\right)$ such that:

$$
\begin{aligned}
\widetilde{K}\left(x_{1: N}, d x_{1: N}^{\prime}\right) & =\left(\tau K_{M}+(1-\tau) K_{E}\right)\left(x_{1: N}, d x_{1: N}^{\prime}\right) \\
K_{M}\left(x_{1: N}, d x_{1: N}^{\prime}\right) & =\prod_{i=1}^{N} K_{i}\left(x_{i}, d x_{i}^{\prime}\right) \\
K_{E}\left(x_{1: N}, d x_{1: N}^{\prime}\right) & =\sum_{i=1}^{N-1} \sum_{l=i+1}^{N} \varepsilon_{i l} K_{E}^{(i l)}\left(x_{i, l}, d x_{i, l}^{\prime}\right) \delta_{x_{-(i, l)}}\left(d x_{-(i, l)}\right)
\end{aligned}
$$

with

$$
\tau, \varepsilon_{i l} \in(0,1) \quad \sum_{i=1}^{N-1} \sum_{l=i+1}^{N} \varepsilon_{i l}=1
$$

and for each $i \in \mathbb{T}_{N} K_{i}$ is an aperiodic, $\lambda$-irreducible Markov kernel with invariant measure $\pi_{i}(x) \lambda(d x)$. Suppose that $K_{j^{*}}$ is uniformly ergodic for one $j^{*} \in \mathbb{T}_{N}$ and for each $i \neq j^{*} \exists \varrho_{i} \in(0, \infty)$ such that $\pi_{i}(x) \leq \varrho_{i} \pi_{j^{*}}(x) \forall x \in E$. Then $\widetilde{K}$ is uniformly ergodic.

Proof. See Appendix 2 for the proof.

Remark 1. The assumption of uniform ergodicity for at least one $K_{j^{*}}$ is not overly restrictive. In many applications, for example, Bayesian analyses with a proper prior and a bounded likelihood, a proposal under an independence kernel with proposal density $q$, where $\pi(x) / q(x) \leq \varrho \forall x \in E$ (see Tierney (1994), Mengersen \& Tweedie (1996)), can be found - for example, where $q$ is the prior density - which ensures 
uniform ergodicity at the cost of being a poor proposal for $\pi$. However, if applied to a related distribution in the population, this proposal may perform quite well. The assumption $\pi_{i}(x) \leq \varrho_{i} \pi_{j^{*}}(x) \forall x \in E$ is quite reasonable and would apply in the framework described here, when $\pi$ is bounded, for the case $j^{*}=N$. Crucially, for small $N$, we can establish that the fast rate of convergence for one of the kernels is propagated through the population (see Section 4.2). We note that the result holds for the case where $K_{M}$ is a mixture of kernels, but have omitted the details for brevity.

Remark 2. The Theorem shows, in simple cases, where we can design uniformly ergodic chains for the target $\pi$ (which are likely to perform well in practice), how to compare population and single chain approaches. In other words we can construct a population sampler which has a faster rate of convergence to $\pi^{*}$ (and hence $\pi$ ) which justifies the increased cost in computation. Additionally, we can investigate

the population kernels, such as $\widetilde{K}$, which are likely to provide good mixing for more complex examples. It should be noted that, due to the limitations of investigating the minorization conditions, it is difficult to use this result when $N$ is large; we discuss this further in Section 4.3 .

\subsection{Impact of the Exchange kernel}

In the following result we show that the exchange kernel, which is reducible, can indeed improve the rate of convergence relative to a parallel MCMC algorithm (no interaction between the chains). To study this phenomenon, we introduce the following mixing condition (M) (e.g. Del Moral (2004)) for Markov kernel $K: E^{N} \times \mathcal{E}^{N} \rightarrow[0,1]: \exists \epsilon>0$ such that $\forall(x, y) \in E^{2 N}$

$$
K(x, \cdot) \geq \epsilon K(y, \cdot)
$$

Also, introduce the set $\mathbb{T}_{N}^{c}=\left\{(i, l): i, l \in \mathbb{T}_{N}, i \neq l\right\}$.

Proposition 2. Assume $K_{M}$ satisfies (M). Then for any initial distribution $\eta$ and $n \geq 1$ :

$$
\left\|\eta\left(K_{M} \circ K_{E}\right)^{n}-\pi^{*}\right\|_{T V} \leq[2(1-\alpha)(1-\epsilon)]^{n}\left\|\eta-\pi^{*}\right\|_{T V}
$$

where $\alpha=\sum_{(i, l) \in \mathbb{T}_{N}^{c}} \varepsilon_{i l}\left[1-\inf _{\left(x_{i}, x_{l}\right) \in E^{2}} \rho_{i, l}\left(x_{i}, x_{l}\right)\right]$ and $\rho_{i, l}\left(x_{i}, x_{l}\right)=\min \left\{1, \frac{\pi_{i}\left(x_{l}\right) \pi_{l}\left(x_{i}\right)}{\pi_{i}\left(x_{i}\right) \pi_{l}\left(x_{l}\right)}\right\}$.

Proof. See Appendix 2 for the proof.

Remark 1. The result provides a sufficient condition to improve upon parallel MCMC, in that it gives a tighter bound on the total variation (which is, under $(\mathrm{M}),(1-\epsilon)^{\frac{3 n}{2}}\left\|\eta-\pi^{*}\right\|_{T V}$ - the factor $3 / 2$ is used to make a fair comparison, in terms of CPU time - for Markov kernel $K_{M}$ ): the exchange probabilities 
are lower-bounded by $1-\frac{(1-\epsilon)^{\frac{1}{2}}}{2}$. Essentially, it is crucial to select a good sequence of densities and use population moves to improve convergence. As noted by a referee, this indicates that if $\epsilon$ is small, that is $K_{M}$ is poorly mixing, the exchange step can help to improve the convergence rate. In addition, if $\forall(x, y) \in E^{2}$,

$(i, l) \in \mathbb{T}_{N}^{c}$ we have $\varrho_{L} \leq \frac{\pi_{i}(x)}{\pi_{l}(y)} \leq \varrho_{U}$ then the lower bound may be achieved if $\frac{\varrho_{L}}{\varrho_{U}} \geq 1-\frac{(1-\epsilon)^{\frac{1}{2}}}{2}$, which suggests that we would require the densities to be similar, to improve convergence rates.

Remark 2. The result is only of use on compact spaces (such as for the finite state space variable selection example of section 4 below), where we are able to lower bound acceptance probabilities and apply the mixing condition (M). However, even on compact spaces, (M) may be difficult to verify for MH kernels due to the rejection probability. We note that this can be circumvented by iterating the kernel.

\section{Example: Bayesian Variable Selection}

We have established the potential theoretical benefits that population MCMC methods offer. A specific example is now studied.

\subsection{Model and Data}

Consider the statistical model:

$$
y_{i}=\gamma_{0}+\sum_{j=1}^{k_{\max }} \vartheta_{j} \gamma_{j} x_{i j}+\varpi_{i}
$$

with $\varpi_{i}$ i.i.d $\mathcal{N}\left(0, \sigma^{2}\right), \vartheta_{j} \in\{0,1\}$, and $\gamma_{j} \in \mathbb{R}$. If we consider the conjugate prior specification: $p\left(\gamma_{0: k} \mid \sigma, k\right)=\mathcal{N}_{k+1}\left(m, \sigma^{2} V\right), p\left(\sigma^{2}\right)=\mathcal{I} \mathcal{G}(a, b)$ (where $\mathcal{I} \mathcal{G}(\cdot, \cdot)$ is the inverse Gamma distribution) and

$$
p\left(\vartheta_{1: k_{\max }}, k\right)=\frac{1}{k_{\max }+1}\left(\begin{array}{c}
k_{\max } \\
k
\end{array}\right)^{-1} \mathbb{I}_{S_{k}}\left(\vartheta_{1: k_{\max }}\right) \quad k=0, \ldots, k_{\max }
$$

(where $S_{k}=\left\{\vartheta_{1: k_{\max }} \in\{0,1\}^{k_{\max }}: \sum_{j=0}^{k_{\max }} \vartheta_{j}=k\right\}$ ) then we can integrate out the parameters $\left(\sigma, \gamma_{0: k}\right)$ and sample from a distribution on a finite state space.

We generated 100 data points from a linear model, with $k_{\max }=8$ (i.e. 256 states). The posterior probability of a null model was 0.55 and 0.33 for the saturated model. This is a typical (but simplified) situation for which a standard MCMC sampler would fail to move around the state space easily.

\subsection{Comparison of Convergence Rates}

To sample from the posterior distribution we use an MCMC algorithm detailed in Denison et al. (2002) page 53 (with modification to the variable selection case). 
Since the state space is finite, it is clear that an ergodic Markov chain with appropriate stationary distribution is uniformly ergodic. To construct an appropriate $\nu$ in (3.1) (i.e. that leads to a large $\epsilon$ ) we used the approach discussed in Chapter 6 of Robert \& Casella (2004) (for example). Let $K_{i j}^{n}(i, j \in E$ ) denote the $n$ step transition probability and suppose that $\inf _{i}\left\{K_{i j}^{n}\right\}>0$ for some $j$, then $\forall j$

$$
K_{i j}^{n} \geq \inf _{l}\left\{K_{l j}^{n}\right\}=\epsilon v_{j}
$$

say, where

$$
v_{j}=\frac{\inf _{i}\left\{K_{i j}^{n}\right\}}{\sum_{l \in E} \inf _{i}\left\{K_{i l}^{n}\right\}} \quad \text { and } \quad \epsilon=\sum_{l \in E} \inf _{i}\left\{K_{i l}^{n}\right\} .
$$

For the algorithm discussed above, we found that the bound on the rate of convergence was reasonably similar for $n_{0}=1000$ to $n_{0}=5000$; we focus upon the pair $\left(1000,3.63 \times 10^{-3}\right)$. To make the analysis computationally comparable to the population algorithm described below, we let 50 applications of this kernel be equivalent to a single step (i.e. this new kernel has $\left(20,3.63 \times 10^{-3}\right)$ as the $\left(n_{0}, \epsilon\right)$ pair).

For a population sampler, suppose we take a single auxiliary distribution:

$$
\pi_{2}\left(\vartheta_{1: k_{\max }}, k \mid x_{1: n}, y_{1: n}\right) \propto L\left(x_{1: n}, y_{1: n} ; \vartheta_{1: k_{\max }}\right)^{\zeta} p\left(\vartheta_{1: k_{\max }}, k\right)
$$

with $\zeta=0.01$ and $L$ the likelihood function. The choice of 0.01 is used for illustration, and is adopted to demonstrate the impact of the usage of a related, but easier to sample, distribution in the population. We concentrate upon a kernel which updates both chains via the MCMC algorithm mentioned above for 10 sweeps, followed by an exchange, then another 10 sweeps (which corresponds to the kernel we sample from, that is, it is a single time step).

It can be shown that the $\left(n_{0}, \epsilon\right)$ pair for the population sampler is $\left(1,6.01 \times 10^{-4}\right)$. This was computed by finding the $\epsilon$ and $\nu$ in the minorization condition for $\pi_{2}$ (as above) and then:

$$
\begin{aligned}
\phi & =\sum_{\left(\vartheta_{1: k_{\max }}, k\right) \in E} \nu\left(\vartheta_{1: k_{\max }}, k\right) \min \left\{1, \frac{\pi_{1}\left(\vartheta_{1: k_{\max }}, k\right)}{\pi_{2}\left(\vartheta_{1: k_{\max }}, k\right) \varrho_{1}}\right\} \\
\varrho_{1} & =\sup _{\left(\vartheta_{1: k_{\max }}, k\right) \in E} \frac{\pi_{1}\left(\vartheta_{1: k_{\max }}, k\right)}{\pi_{2}\left(\vartheta_{1: k_{\max }}, k\right)}
\end{aligned}
$$

the constant in the minorization condition is then $\epsilon^{2} \phi$.

The bound on total variation distance, $R$, suggests a much faster rate of convergence for the population algorithm: $M_{0.01}=25326$ (the number of iterations to achieve a bound on the total variation distance less than 0.01) for the vanilla algorithm and $M_{0.01}=7660$ for the population algorithm, that is, it is significantly faster.

This example demonstrates that a simple extension of the original algorithm to include an auxiliary distribution that provides a good proposal (for the original target) allows efficient movement around the 
state space. That is, it reiterates the point in remark 1 to Theorem 1, that the fast convergence rate of one of the chains is propagated through the system.

\subsection{Summary}

A point of interest, raised by a referee, is that we found as we increased $N$ we found it difficult to find a Markov kernel that could improve upon rate of convergence to stationarity when $N=2$. This can be explained as follows; suppose, for illustration, we run $N$ parallel chains with the same (marginal) invariant measure $\pi(x) \lambda(d x)$ with kernel $K_{M} \circ K_{E} \circ K_{M}$ in the proof of Theorem 1 Assume that all the mutation kernels, except one, are strongly aperiodic $\left(n_{0}=1\right)$ and mix almost perfectly (that is, are uniformly ergodic with $\epsilon \approx 1$ ). Then we can easily establish that the constant in the minorization condition is $\epsilon^{N} \rightarrow 0$ as $N \rightarrow \infty$, so that $R \approx 1$. This establishes two points:

- Firstly, that continually extending the state-space will inevitably lead to slower convergence of the Markov chain, unless very efficient population moves may be constructed. That is, we should not naively extend the state-space and expect our Markov chain to converge more quickly; unlike convergence of particle algorithms (Del Moral, 2004), there is not necessarily an improved convergence property as $N \rightarrow \infty$. This is an open problem for future research.

- Secondly, that in cases where $R$ is not informative on the rate of convergence, it may be more beneficial to investigate other properties of the Markov kernel, such as the spectral gap. Such an analysis is likely to be far more involved than discussed here; see Madras \& Zheng (2003) for example.

\section{Population Moves for the Mixture Example}

Now that we have established, for difficult problems, that population methods can lead to faster convergence, we discuss how to implement population moves for our mixture example (Section 2.3). Our notation is such that $\theta_{i}=\left(\eta^{i}, w^{i}, \Psi^{i}, k_{i}\right), i \in \mathbb{T}_{N}$ and $\left(\eta^{i}, w^{i}\right)$ refers to all of the component specific parameters and weights for chain $i$. In our tempering approach (i.e. $\pi^{\zeta_{i}}$ ), we will temper the likelihood terms only, rather than the full posterior, to avoid any integrability problems. We now proceed to combine several MCMC methods to improve the mixing ability of the chain.

\subsection{Exchange Moves}

An exchange move is used to swap information between two different parallel tempered chains. Our strategy is as follows: at iteration $t$ we select two adjacent chains (in terms of the temperature parameter) uniformly at random and propose to swap their values. In order to achieve a reasonable interaction between 
the chains, the temperature ladder is set so that this move is accepted about half of the time (Liu, 2001); see Section 6 for further discussion.

One way to improve this move is to use the delayed rejection method, as suggested by Green \& Mira (2001). At iteration $t$, we select any two chains $i_{1}$ and $i_{2}$ to swap, accepting or rejecting with the usual Hastings ratio, that is, with probability

$$
\rho_{1}\left(\theta_{1: N}, \theta_{1: N}^{\prime}\right)=\min \left\{1, \frac{\pi_{i_{1}}\left(\theta_{i_{2}}\right) \pi_{i_{2}}\left(\theta_{i_{1}}\right)}{\pi_{i_{1}}\left(\theta_{i_{1}}\right) \pi_{i_{2}}\left(\theta_{i_{2}}\right)}\right\}
$$

where the labelling of the chains is with respect to the current state of the chain and $\theta_{1: N}^{\prime}$ denotes the new configuration of chains. If this is rejected, we select two adjacent chains $i_{3}$ and $i_{4}$ to swap, denoting this configuration $\theta_{1: N}^{\prime \prime}$. To ensure reversibility with respect to the target, as part of the delayed rejection method, we construct a pseudo move which consists in starting from the second stage proposed state $\theta_{1: N}^{\prime \prime}$, proposing to move to $\theta_{1: N}^{*}$ (which swaps $\theta_{i_{1}}^{\prime \prime}$ and $\left.\theta_{i_{2}}^{\prime \prime}\right)$ and rejecting it with probability $\rho_{1}\left(\theta_{1: N}^{\prime \prime}, \theta_{1: N}^{*}\right)$. The second stage move is accepted with probability

$$
\rho_{2}\left(\theta_{1: N}, \theta_{1: N}^{\prime \prime}\right)=\min \left\{1, \frac{\pi_{i_{3}}\left(\theta_{i_{4}}\right) \pi_{i_{4}}\left(\theta_{i_{3}}\right)\left\{1-\rho_{1}\left(\theta^{\prime \prime}, \theta^{*}\right)\right\}}{\pi_{i_{3}}\left(\theta_{i_{3}}\right) \pi_{i_{4}}\left(\theta_{i_{4}}\right)\left\{1-\rho_{1}\left(\theta, \theta^{\prime}\right)\right\}}\right\} .
$$

This move allows for increased interaction within the population. At the first stage, we allow any pair of chains to be swapped, thus if a state of a chain at a high temperature is consistent with one of the distributions at a lower temperature, it is allowed to quickly jump down the ladder.

\subsection{Crossover Moves}

Liang \& Wong (2001) employ various crossover moves in an evolutionary MC algorithm; the objective is to increase the interaction within the population. In our algorithm, we use two move types:

\section{Variable dimension crossover}

To construct a move likely to have high acceptance probability in the mixture model, we begin by reassigning mixture component labels in each chain so they satisfy an ordering constraint on the weights, in order approximately to match the labels of components in different chains. When in state $\theta$, we select a variable dimension crossover with probability $v(\theta)$;

$$
v(\theta)= \begin{cases}1 & \text { if } k_{i} \neq k_{j} \text { for some } i \neq j \\ 0 & \text { otherwise. }\end{cases}
$$

Note the case $v(\theta)=0$ corresponds to a 'do nothing' move. We select a pair of chains with differing dimension with probability inversely proportional to the squared difference between the dimensions. We then propose the new state of the population members, by swapping $k$ 's and the weights. We take the 
lowest weighted component specific parameters of the higher dimensional chain to the lower dimensional chain, i.e. if $k_{i_{1}}>k_{i_{2}}$ for the selected chains $i_{1}, i_{2}$ we propose:

$$
\begin{aligned}
\eta_{i_{1}}^{\prime} & =\left(\eta_{k_{i_{1}}-k_{i_{2}}}^{i_{1}}, \ldots, \eta_{k_{i_{1}}}^{i_{1}}\right) \\
\eta_{i_{2}}^{\prime} & =\left(\eta_{1}^{i_{1}}, \ldots, \eta_{k_{i_{1}}-k_{i_{2}-1}}^{i_{1}}, \eta_{1}^{i_{2}}, \ldots, \eta_{k_{i_{2}}}^{i_{2}}\right)
\end{aligned}
$$

where $\eta_{j}^{i_{1}}$ denotes an element of $\eta^{i_{1}}$. The acceptance probability is easily calculated and thus omitted. After the move has been accepted or rejected (or the do nothing move) we propose (and accept) a random permutation (all permutations have uniform probability of being proposed) of the labels of the parameters (of all the chains). This final permutation ensures invariance with respect to the target.

\section{Fixed Dimension Crossover}

We begin by reassigning the mixture component labels in each chain by ordering on the first dimension of the means. When in state $\theta$, we select a fixed dimension crossover with probability 1 , if it can be selected (i.e. there are at least two chains with the same dimensionality) otherwise we select a 'do nothing' move. Select a pair of chains $\left(i_{1}, i_{2}\right)$ with the same dimensionality, with probability

$$
p\left(i_{1}, i_{2} \mid \theta\right) \propto\left|\zeta_{i_{1}}-\zeta_{i_{2}}\right|^{-1} \mathbb{I}_{k_{i_{1}}=k_{i_{2}}}
$$

We select a position $j=1, \ldots, k_{i_{1}}-1$ to crossover, this selection made with probability proportional to $1 / j$ and switch all component specific parameters to the left of $j$ inclusive (note that if the identifiability constraint is not satisfied in the proposed state of the chain we immediately reject) and accept/reject on the basis of the Hastings ratio. After the accept/reject (or the do nothing move) decision has been made, we again propose a random permutation of the labels of the parameters.

\subsection{Snooker Jumps}

One of the most important ways we can use the information in the population is by targeting variable dimension jumps by using another chain. This idea is linked to the snooker algorithm of Gilks et al. (1994) and is performed in the following way. When in state $\theta$, we select a birth with probability $b(\theta)$, where

$$
b(\theta)= \begin{cases}1 & \text { if } k_{i}=1 \forall i \\ 0 & \text { if } k_{i}=k_{\max } \forall i \\ 1 / 2 & \text { otherwise }\end{cases}
$$

then select a chain (the current point $\theta_{c}$ ) for which a birth is possible (let $m_{b}(\theta)$ be the number of chains such that a birth can occur when in state $\theta$ ) with uniform probability, and select an anchor point $\left(\theta_{a}\right)$ with 
probability inversely proportional to the absolute value of the difference between the inverse temperatures. We then generate $w \sim \mathcal{B} e\left(1, k_{c}\right)$, with $\mathcal{B} e(\cdot, \cdot)$ the beta density, and draw a new $\mu, \Lambda$ pair from:

$$
q(\mu, \Lambda)=\sum_{j=1}^{k_{a}} \bar{h}\left(\eta_{j}\right) \mathcal{N}_{r}\left(\mu_{j}^{a}, \sigma\right) \mathcal{I} \mathcal{W}\left(2 r+3, \Lambda_{j}^{a}\right)
$$

where

$$
\bar{h}\left(\eta_{j}\right) \propto \frac{1}{k_{c}} \sum_{l=1}^{k_{c}} h\left(\left(\mu_{j}^{a}, \Lambda_{j}^{a}\right),\left(\mu_{l}^{c}, \Lambda_{l}^{c}\right)\right)
$$

and $h(\cdot, \cdot)$ is the Mahalanobis distance. We then perform the rest of the move as for the birth in Appendix 1. In the death move, we perform much the same as for Appendix 1, except we select a current point with probability $1 / m_{d}(\theta)$ (where $m_{d}(\theta)$ is the number of chains for which a death can occur when in state $\theta$ ) and (redundantly) select an anchor point (which is used in the reverse birth). The birth move is accepted with probability $\min \{1, A\}$ with:

$$
\begin{aligned}
A= & \frac{p\left(y_{1: n} \mid \eta^{\prime}, w_{1: k_{c}}^{\prime c}, k_{c}+1\right)^{\zeta_{c}} p(\mu) p(\Phi) p\left(k_{c}+1\right)}{p\left(y_{1: n} \mid \eta^{c}, w_{1: k_{c}-1}^{c}, k_{c}\right)^{\zeta_{c}} p\left(k_{c}\right)} B\left(k_{c} \delta, \delta\right)^{-1} w^{\delta-1}(1-w)^{k_{c}(\delta-1)} \frac{\left(k_{c}+1\right) !}{k_{c} !} \\
& \times \frac{d\left(\theta^{\prime}\right) m_{b}(\theta)}{\left(k_{c}+1\right) b(\theta) m_{d}\left(\theta^{\prime}\right)} \frac{(1-w)^{k_{c}-1}}{\mathcal{B} e\left(w ; 1, k_{c}\right) q(\mu, \Phi)}
\end{aligned}
$$

where $\Phi$ is the Cholesky decomposition of $\Lambda$ (see Appendix 1 for details), $p\left(y_{1: n} \mid \eta^{c}, w_{1: k_{c}-1}^{c}, k_{c}\right)^{\zeta_{c}}$ is the tempered likelihood (for the current point), $B(\cdot, \cdot)$ is the beta function and $\mathcal{B} e(x ; \cdot, \cdot)$ is the beta density evaluated at $x$. The objective of this move is to propose new component-specific parameters which are likely to be consistent with the data, but are markedly different from the current components. It also provides an adaptive element to the birth proposal, as it relies on current information, in the population, that is being continuously updated.

\subsection{Constrained Sampling}

One aspect of population-based simulation that is apparent is the need to maintain diversity of the population (as in sequential Monte Carlo - see Del Moral et. al. (2006)). In many cases for which it is difficult to traverse the state space, it is often the case that the chains at lower temperatures can become trapped (stuck in local modes) as for single chain MCMC methods, and this may lead to inaccurate Monte Carlo estimates of quantities of interest. To avoid this problem, we propose to constrain some of the members of the population, that is, for some subset $\mathbb{T}_{l: N}(l \geq 2)$, and $i \in \mathbb{T}_{l: N}, \pi_{i}$ is a density constrained to $E_{i} \subset E$. In the setting of trans-dimensional problems, a natural choice of sets $E_{i}$ may be selected with respect to the model dimension (e.g. $E_{i}=\bigcup_{k \in \mathcal{K}_{i}}\left(\{k\} \times E^{k}\right)$ for $\mathcal{K}_{i} \subset \mathcal{K}$ and $\left.i \in \mathbb{T}_{l: N}\right)$. In general, choosing an appropriate $E_{i}$ is challenging; see Atchadé \& Liu (2004) (unpublished technical report) for some discussion. We remark that, in the example in Section [6 this technique will prove to be very important. 


\subsection{The Algorithm}

To sample from the augmented distribution we use the algorithm below; we use the genetic algorithm terminology of Liang \& Wong (2001).

0. Initialise the chain $\theta$.

For $t=1, \ldots, M$ sweep over the following:

1. Mutation. Select a chain $i \in\{1, \ldots, N\}$ with probability $\tau_{i}$ and then perform one sweep of the reversible jump algorithm in Appendix 1 for this chain (with appropriate modification for constrained targets).

2. Make a random choice between performing steps 3 or 4 .

3. Crossover. Propose a variable dimension crossover move with probability $1 / 2$, else propose a fixed dimension crossover.

4. Snooker Jump. Propose a birth with probability $b(\boldsymbol{\theta})$, else propose a death.

5. Exchange. Perform the delayed rejection exchange move.

Note that, for constrained chains, we only allow them to be involved in fixed dimensional crossovers and a special exchange move that augments step 5; we propose to exchange a constrained and non-constrained chain, selecting the move only if such a move may be performed (that is $k_{i} \in \mathcal{K}_{j}$ and $k_{j} \in \mathcal{K}_{i}$ for $i \neq j$ ). All selections are made with uniform probability and no delayed rejection is used.

\section{Gene expression example revisited}

\subsection{Specification of Simulation Parameters}

Population Size : To run the population algorithm in Section 5.5] we used $N=25$ with 5 chains constrained. We recommend a large population size in general (although the discussion in Section 4.3 should be considered), so that results are reasonably similar for separate runs of the algorithm. See Jasra, Stephens \& Holmes (2006) (unpublished technical report, available from http://stats.ma.ic.ac.uk/das01/) for more guidance.

Temperature Parameters: For the main population (the unconstrained chains) the following inverse temperatures were selected:

$$
\begin{aligned}
\zeta_{1} & =1 \\
\zeta_{i} & =\zeta_{i-1}-\varsigma \varphi^{i-1} i \in \mathbb{T}_{2: 20}
\end{aligned}
$$

for constants $\varsigma>0, \varphi>1$. We selected $\varsigma=10^{-6}, \varphi=1.85\left(\zeta_{20}=0.74\right)$. Our choice of cooling schedule, and population size was based upon pilot tuning. We selected a slowly decreasing sequence of $\zeta$ 's since 
we observed a poor acceptance rate for the exchange move for distributions that were further away from each other, as expected. We found that the inverse temperature at which the reversible jump algorithm performed best (that is, reasonable acceptance rates along with regions of high support that were similar to the target) was $\zeta=0.75$; thus we attempted to include a distribution with this temperature. We note that we need to be careful when specifying temperatures, since for low temperatures (low depending on the problem at hand) the distribution starts to favour dimensionalities that are small, although this may be alleviated by specifying priors for $k$ which penalise small values. For this particular problem, we spent at least 2-3 hours in tuning the parameters; a more automatic procedure may be implemented - see below.

The term 'reasonable acceptance rate' deserves a quantitative definition. A useful criterion put forward by Iba (2001) is that the expected (wrt target) log Hastings ratio of the exchange probability is equal to 1. This in turn means that, approximately, the algorithm will accept the exchange about half the time. We remark that such an approach may be used to provide an automatic temperature selection and will vastly reduce the time spent on tuning the algorithm (as noted by a referee this aspect should be taken into account in the computational cost and comparison of the algorithm). We refer the reader to Iba (2001) for the details and to Goswami \& Liu (2006) (unpublished technical report) for an alternative approach.

Constrained Targets: To select the $E_{i}, i \in \mathbb{T}_{21: 25}$ we used a pilot simulation. We ran the algorithm with $N=25$ and the inverse temperature parameters discussed above, only 9 chains in the main population and 16 constrained chains (given inverse temperature parameter 0.999). We selected the subspaces $E_{i}$ with respect to the dimensionality, that is we had 10 chains constrained to lie $k \in \mathbb{T}_{1: 2}, \ldots, \mathbb{T}_{19: 20}$ then six other chains constrained to lie in $k \in \mathbb{T}_{3: 6}, \mathbb{T}_{6: 9}, \mathbb{T}_{9: 12}, \mathbb{T}_{12: 15}$ and $\mathbb{T}_{15: 18}$. This was adopted in order to determine whether there was any support outside $\mathbb{T}_{3: 11}$ found in Section 2.3 Based upon a short run, the five constrained chains were taken as $\mathbb{T}_{2: 4}, \mathbb{T}_{4: 6}, \mathbb{T}_{5: 7}$ and $\mathbb{T}_{7: 9}, \mathbb{T}_{9: 11}$. The idea of the pilot tuning is to avoid wasting CPU time on population members constrained to lie in areas of support that have low density with respect to the original target density. Additionally, the constrained chains need to be able to interact with the main population and the preliminary tuning allows us to make this choice. The inverse temperature parameters for the constrained chains were 0.999 , since we seek to maintain diversity with respect to the population at colder temperatures. For further discussion in the setting of partitions, especially in the context of reversible jump, see Atchadé \& Liu (2004) (unpublished technical report).

The algorithm in Section 5.5 was run for 1 million sweeps which took approximately $9 \frac{1}{2}$ hours (code is available on request from the first author). A minor modification to algorithm was made: that if a crossover move was selected, we also propose an exchange for the chain of interest. 


\subsection{Comparison with Vanilla Sampler}

The improvement over the vanilla sampler is substantial, on average, the chain of interest took 6.75 sweeps to jump between a mixture model with less than 4 components to a mixture with more than 6 .

The observed inability of the vanilla reversible jump algorithm to move around the state space (from $k \in \mathbb{T}_{3: 5}$ to $\left.k \in \mathbb{T}_{8: 11}\right)$ is not present for the population sampler, since it may represent both parts of the space simultaneously. Note that, due to the complexity of the target, we cannot claim that the sampler has converged; there may be regions of high posterior density that are still unexplored.

It is apparent, from simulations, that despite the substantially improved performance when compared to the vanilla sampler, the sampler has missed the region $k \in\{8,9,10\}$. This is due to the reasons discussed earlier, and demonstrates that constrained chains can help guard against such problems.

The effective sample size (ESS) is a standard measure of the relative efficiency of an MCMC sampler (see Liu (2001) for full definition). For our population algorithm, the ESS for $k$ was 59745 (60000 samples, using a lag of 10 in the autocorrelation calculation), compared with 2998 and 3591 for both vanilla algorithms. Taking

$$
\mathrm{E}=2 \frac{\mathrm{ESS}_{\mathrm{pop}}}{M_{\mathrm{pop}} T_{\mathrm{pop}}} /\left[\frac{\mathrm{ESS}_{\mathrm{van}_{1}}}{M_{\mathrm{van}_{1}} T_{\mathrm{van}_{1}}}+\frac{\mathrm{ESS}_{\mathrm{van}_{2}}}{M_{\mathrm{van}_{2}} T_{\mathrm{van}_{2}}}\right]
$$

where the subscripts refer to the population, vanilla algorithm runs 1 and 2 (the chains run in Section 2.3) and chains respectively, $M$ is the sample size and $T$ is the $\mathrm{CPU}$ time, we obtain $\mathrm{E}=2.07$ (we note that similar conclusions are drawn when taking into account tuning time). Therefore there is little contest between using population-based reversible jump and the vanilla counterpart for this example; the population approach is far superior (note that all coincidental simulation parameters are the same between algorithms).

\subsection{The Efficiency of Sampler Moves}

The exchange move was accepted $44 \%$ of the time at the first stage and $75 \%$ at the second. This indicates that delayed rejection helps to ensure that the algorithm is constantly swapping information between the chains; for $86 \%$ of the sweeps there is at least one exchange.

The snooker and variable dimension crossover moves have acceptance rate less than $1 \%$. That this occurs is to be expected. Liang \& Wong (2001) report fairly small acceptance rates for their crossover moves; our rates are smaller as our algorithm operates upon are a more complex space. Our experience with the snooker and variable dimension crossover in more simple examples, is that they are generally not worth the extra coding effort given their performance. However, we were satisfied with the fixed dimensional crossover which was accepted $2.9 \%$ of the time. The snooker birth is accepted more often than 
the standard birth, but the reverse snooker death move is rarely accepted (c.f. a birth move with a proposal that has low variance). Hence the move is less successful overall than the standard birth. The variable dimension move acceptance rates (averaged over all chains) were still below 1\%, with the split/combine move being less effective.

Overall, our recommendation is that the exchange (with delayed rejection) and fixed dimensional crossover are implemented. This is in conjunction with constrained targets.

\subsection{Comparison with Simulated Tempering}

A more appropriate single chain sampler to compare with the population method is a simulated tempering algorithm; see Hodgson (1999) for an example of another variable dimension simulated tempering algorithm. Here the target distribution is:

$$
\pi\left(\theta, \zeta, k \mid y_{1: n}\right) \propto p\left(y_{1: n} \mid \theta, k\right)^{\zeta} p(\theta, k) p(\zeta)
$$

that is, $\zeta \in Z$ is stochastic with $Z$ finite and pseudo prior $p(\zeta)$ (in this example we set the prior as opposed to constructing one adaptively; see Geyer \& Thompson (1995)). Note that our formulation does not require any normalization constants to be known, although such an algorithm will allow us to find a 'good' pseudo prior; see Geyer \& Thompson (1995).

To sample from (6.3) we use the reversible jump algorithm in Appendix 1, conditional upon $\zeta$, and to update $\zeta$ a delayed rejection move was adopted: propose a temperature uniformly at random from $Z$ and accept or reject with the Hastings ratio - if rejected, select an adjacent temperature and perform a pseudo move that selects to move from the proposed temperature at the second stage to the proposed temperature at the first stage.

Using some trial simulations we were unable to find a pseudo prior so that for a reasonable number of temperatures (e.g. 25), the algorithm could jump between the target and the inverse temperature 0.75. As a result, for any sensible number of distributions, the performance of this approach only slightly improves over the vanilla algorithm. An example of a run of the simulated tempering algorithm was setting $p(\zeta=$ $\left.\zeta_{i}\right) \propto 1 / i$, with $\zeta_{1}=1$ and having a difference of $1 \times 10^{-4}$ between each temperature. We found, with $|Z|=25$, that the algorithm only visited the distribution of interest $10 \%$ of the time in a run of 250000 sweeps.

In this Section, it was seen that simulated tempering has been difficult to set up, so that it can be operated efficiently; see Atachdé \& Liu (2004) (unpublished technical report) for a more automatic procedure. In addtion, see Zheng (2003) for a theoretical comparison of population MCMC and simulated tempering. 


\section{Discussion}

To summarize, in our experience a vanilla reversible jump algorithm often fails to explore the support of a multimodal model space. We introduced population-based reversible jump MCMC and gave some theoretical justification for why these methods can be preferable to standard MCMC methods. In addition, it was demonstrated that population-based reversible jump is a means to improving variable dimension simulation. Overall, our method helps open up the possibility of fully Bayesian analyses in problems for which simulation is prohibitively slow. Note that the basic algorithm (without population moves) can easily be coded given a vanilla sampler. Therefore population MCMC provides a simple way to check the performance of MCMC algorithms.

One of the problems of our approach is the limited amount of success of our crossover moves. Whilst this was observed for simpler problems in Liang \& Wong (2001), we would still hope that the population can provide more information when proposing moves. General guidelines for constructing constrained subspaces of $E$ and finding efficient ways to make them interact with the population is an important area for further investigation. There are many potential methodological extensions that may be considered.

Firstly, to combine our approach with adaptive MCMC methods (see Andrieu \& Moulines (2006) and Chauveau \& Vandekerkhove (2002) in the population context). This is likely to be superior to standard adaptive algorithms, since there is more information to update proposals. Furthermore, there is more information in terms of where the chain has not been, i.e. we may search (fewer) regions of the support of $\pi$ for states with high density.

Secondly, we may consider combining some of our ideas with many recent stochastic simulation algorithms. For example, we might use the constrained chains in a similar context to the equi-energy sampler of Kou et al. (2006). In the trans-dimensional case, the energy rings could be replaced with dimension rings. This is likely to produce a highly diverse sample with respect to the dimensionality.

\section{Acknowledgement}

The first author was supported by an PhD EPSRC Studentship. We thank two referees and an associate editor for comments which lead to a substantial improvement in the content of the paper. The first author acknowledges many useful conversations with Nick Heard, Matthew Gander and Zhicheng Zhang. We also thank Adam Johansen and Tso-Jung Yen for some comments on earlier versions of the paper.

\section{Appendix 1: Reversible Jump Sampler}

The vanilla sampler used in Section 2.3 is now outlined. 
One of the drawbacks of the model we have selected is the need for $\Lambda$ to be positive definite. As a result, moves in MCMC simulation will be difficult to construct such that this constraint is satisfied. To deal with this problem we consider the Cholesky decomposition $\Phi$ (see Dellaportas \& Papageorgiou (2006) for an analysis using the spectral decomposition). That is, $\Lambda=\Phi \Phi^{\prime}$ where $\Phi$ is lower triangular with positive diagonal elements (recall the Jacobian is $2^{r} \prod_{l=1}^{r} \phi_{l l}^{r-l+1}$ ). Our RJMCMC algorithm is as follows (all moves are Metropolis-Hastings steps unless otherwise stated).

Firstly the fixed dimensional moves. The component specific means $\left(\mu_{j}\right)$ and component specific lower triangular part of $\Phi_{j}$ are both updated via an additive cauchy random walk, independent in each dimension. The component specific diagonals of $\Phi_{j}$ are updated via a multiplicative log-normal random walk, independent in each dimension. The weights are proposed using an additive normal random walk on the logit scale. Finally, $\Psi$ is generated using a Gibbs kernel; the full conditional is $\mathcal{W}\left(2\left(g+k \alpha^{\prime}\right),\left(2 h+2 \sum_{j=1}^{k} \Lambda_{j}^{-1}\right)^{-1}\right)$.

Secondly a birth/death of a component, largely following Richardson \& Green (1997). Briefly, we draw a new $\mu$ and $\Phi$ from the prior and $w \sim \mathcal{B} e(1, k)$, setting the new weights as $\left(w_{1}(1-w), \ldots, w_{k}(1-w), w\right)$, selecting the move with probability $b_{k}$ (when in state $k$ ). The death, selected with probability $d_{k}$, is performed by selecting a component to die with uniform probability and inverting the jump function.

Finally, a split/combine of a component. We select a split with probability $s_{k}$ and choose a component $j^{*}$ uniformly at random to split into components labelled as $\left(j_{1}, j_{2}\right)$. The split requires the following actions:

(i) Split the weight by drawing $u_{1} \sim \mathcal{B} e(\gamma, \gamma)$ and set

$$
\begin{aligned}
& w_{j_{1}}=u_{1} w_{j^{*}} \\
& w_{j_{2}}=\left(1-u_{1}\right) w_{j^{*}} .
\end{aligned}
$$

(ii) Split the mean vector by drawing $u_{1(2)}, \ldots, u_{r(2)} \sim \mathcal{N}\left(0, \sigma_{\mu}\right)$ and take

$$
\begin{aligned}
& \mu_{l\left(j_{1}\right)}=\mu_{l\left(j^{*}\right)}+u_{l(2)} \\
& \mu_{l\left(j_{2}\right)}=\mu_{l\left(j^{*}\right)}-u_{l(2)} .
\end{aligned}
$$

(iii) Split the off diagonals of $\Phi$ by drawing $u_{21(3)}, \ldots, u_{r(r-1)(3)} \sim \mathcal{N}\left(0, \sigma_{\phi}\right)$ and take

$$
\begin{aligned}
& \phi_{l m\left(j_{1}\right)}=\phi_{\operatorname{lm}\left(j^{*}\right)}+u_{l m(3)} \\
& \phi_{l m\left(j_{2}\right)}=\phi_{l m\left(j^{*}\right)}-u_{l m(3)}
\end{aligned}
$$

where $l=2, \ldots, r, m=1, \ldots, l-1$.

(iv) Split the diagonals of $\Phi$ by drawing $u_{11(3)}, \ldots, u_{r r(3)} \sim \mathcal{L N}(0, \sigma)$ and take

$$
\phi_{l l\left(j_{1}\right)}=\frac{\phi_{l l\left(j^{*}\right)}}{u_{l l(3)}} \quad \phi_{l l\left(j_{2}\right)}=\phi_{l l\left(j^{*}\right)} u_{l l(3)} .
$$


In order to combine we select the move with probability $c_{k}$ and invert the jump function above. We note that due to the symmetry constraint imposed on the jump function it does not matter which way we combine the components (see Cappé et al.(2003) for details on this). We choose two components to combine, when in state $k$, with probability inversely proportional to the Mahalanobis distance between them, that is:

$$
p_{k}\left(j_{1}, j_{2}\right) \propto\left[\left(\mu_{j_{1}}-\mu_{j_{2}}\right)^{\prime} \Lambda_{j_{1}}^{-1}\left(\mu_{j_{1}}-\mu_{j_{2}}\right)+\left(\mu_{j_{2}}-\mu_{j_{1}}\right)^{\prime} \Lambda_{j_{2}}^{-1}\left(\mu_{j_{2}}-\mu_{j_{1}}\right)\right]^{-1}
$$

The split in state $k$ is accepted with probability $\min \{1, A\}$ where

$$
\begin{aligned}
A= & (\text { likelihood ratio }) \frac{p\left(\Phi_{j_{1}}\right) p\left(\Phi_{j_{2}}\right)}{p\left(\Phi_{j^{*}}\right)} \frac{p\left(\mu_{j_{1}}\right) p\left(\mu_{j_{2}}\right)}{p\left(\mu_{j^{*}}\right)} B(k \delta, \delta)^{-1}\left(w_{j^{*}} u_{1}\left(1-u_{1}\right)\right)^{\delta-1} \times \\
& \frac{p(k+1)}{p(k)} \frac{(k+1) !}{k !} \frac{k c_{k+1} p_{k+1}\left(j_{1}, j_{2}\right)}{s_{k}} \frac{|J|}{2 q_{1}\left(u_{1}\right) q_{2}\left(u_{2}\right) q_{3}\left(u_{3}\right)}
\end{aligned}
$$

where $|J|$ is the Jacobian:

$$
|J|=2^{\frac{r(r+3)}{2}} w_{j^{*}} \prod_{l=1}^{r} \frac{\phi_{l l\left(j^{*}\right)}}{u_{l l(3)}}
$$

and obvious notation for the prior and proposal densities.

The algorithm is performed in a deterministic sweep over all fixed dimension moves followed by a random choice of birth/death or split/merge. The particular trans-dimensional move is selected with uniform probability (assuming we allow a move, i.e. no birth or split when $k=k_{\max }$ or death or combine when $k=1)$.

\section{Appendix 2: Proofs}

Proof of Theorem 11. Our strategy is to show that $\widehat{K}^{N-1}\left(x_{1: N}, A\right)=\left(K_{M} \circ K_{E} \circ K_{M}\right)^{N-1}\left(x_{1: N}, A\right)(A \in$ $\left.\mathcal{E}^{N}\right)$ is a uniformly ergodic Markov kernel. Then to prove $\widetilde{K}^{3(N-1)}$ is uniformly ergodic, we may use the fact that $\widetilde{K}^{3(N-1)}\left(x_{1: N}, A\right) \geq \tau^{2(N-1)}(1-\tau)^{N-1} \widehat{K}^{N-1}\left(x_{1: N}, A\right)$ (proved below). We begin by proving the case $N=2$ and then use an induction on $N$. The strategy of the proof is to use the uniform ergodicity of $K_{j^{*}}$ (which we take to be $K_{N}$ ) and the acceptance of an exchange move. We assume $n_{0}=1$ as the proof can be extended to the case $n_{0}>1$ with only notational complications. We denote $x_{i}^{(l)}$ as the value of $x_{i}$ after $l$ steps.

We first establish $\widetilde{K}^{3(N-1)}\left(x_{1: N}, A\right) \geq \tau^{2(N-1)}(1-\tau)^{N-1} \widehat{K}^{N-1}\left(x_{1: N}, A\right), A \in \mathcal{E}^{N}$. Consider $\widetilde{K}^{3}$, then:

$$
\begin{aligned}
\widetilde{K}^{3}\left(x_{1: N}, A\right) & =\int_{E^{N}} \widetilde{K}\left(x_{1: N}, d x_{1: N}^{(1)}\right) \int_{E^{N}} \widetilde{K}\left(x_{1: N}^{(1)}, d x_{1: N}^{(2)}\right) \widetilde{K}\left(x_{1: N}^{(2)}, A\right) \\
& \geq \int_{E^{N}} \widetilde{K}\left(x_{1: N}, d x_{1: N}^{(1)}\right) \int_{E^{N}} \widetilde{K}\left(x_{1: N}^{(1)}, d x_{1: N}^{(2)}\right) \tau K_{M}\left(x_{1: N}^{(2)}, A\right)
\end{aligned}
$$


where we have applied Chapman-Kolmogorov and used $\widetilde{K}\left(x_{1: 2}, \cdot\right) \geq \tau K_{M}\left(x_{1: N}, \cdot\right) \forall x_{1: N} \in E^{N}$. We then have

$$
\widetilde{K}^{3}\left(x_{1: N}, A\right) \geq \tau^{2}(1-\tau) \int_{E^{N}} K_{M}\left(x_{1: N}, d x_{1: N}^{(1)}\right) \int_{E^{N}} K_{E}\left(x_{1: N}^{(1)}, d x_{1: N}^{(2)}\right) K_{M}\left(x_{1: N}^{(2)}, A\right)
$$

which corresponds to selecting a mutation followed by an exchange and then followed again by another mutation. This clearly follows for $\widetilde{K}^{3(N-1)}\left(x_{1: N}, A\right)$, by the argument above:

$$
\begin{aligned}
\widetilde{K}^{3(N-1)}\left(x_{1: N}, A\right) & \geq \tau^{2}(1-\tau) \int_{E^{N}} \widetilde{K}^{3(N-2)}\left(x_{1: N}, d x_{1: N}^{3(N-2)}\right) \widehat{K}\left(x_{1: N}^{3(N-2)}, A\right) \\
& \geq \tau^{4}(1-\tau)^{2} \int_{E^{N}} \widetilde{K}^{3(N-3)}\left(x_{1: N}, d x_{1: N}^{3(N-3)}\right) \widehat{K}^{2}\left(x_{1: N}^{3(N-3)}, A\right)
\end{aligned}
$$

and we may thus apply the argument above, recursively, to demonstrate the result. We now drop the $\tau^{2}(1-\tau)$ and prove uniform ergodicity of $\widehat{K}$.

Let $N=2, A=A_{1} \times A_{2} \in \mathcal{E} \otimes \mathcal{E}$. Using the fact that $K_{M}\left(x_{1: 2}, \cdot\right)=K_{1}\left(x_{1}, \cdot\right) K_{2}\left(x_{2}, \cdot\right)$ and applying the minorization condition for $K_{2}\left(x_{2}^{(2)}, A_{2}\right)$, the modified equation (7.4) becomes

$$
\begin{aligned}
\widehat{K}\left(x_{1: 2}, A\right) \geq & \int_{E^{2}} K_{1}\left(x_{1}, d x_{1}^{(1)}\right) K_{2}\left(x_{2}, d x_{2}^{(1)}\right) \times \\
& \int_{E^{2}} \delta_{x_{1}^{(1)}}\left(d x_{2}^{(2)}\right) \delta_{x_{2}^{(1)}}\left(d x_{1}^{(2)}\right) \min \left\{1, \frac{\pi_{1}\left(x_{2}^{(1)}\right) \pi_{2}\left(x_{1}^{(1)}\right)}{\pi_{1}\left(x_{1}^{(1)}\right) \pi_{2}\left(x_{2}^{(1)}\right)}\right\} \times \\
& K_{1}\left(x_{1}^{(2)}, A_{1}\right) \epsilon \nu\left(A_{2}\right)
\end{aligned}
$$

where we have ignored the rejection of an exchange move. Since

$$
\min \left\{1, \frac{\pi_{1}\left(x_{2}\right) \pi_{2}\left(x_{1}\right)}{\pi_{1}\left(x_{1}\right) \pi_{2}\left(x_{2}\right)}\right\} \geq \min \left\{1, \frac{\pi_{1}\left(x_{2}\right)}{\pi_{2}\left(x_{2}\right) \varrho_{1}}\right\} \forall\left(x_{1}, x_{2}\right) \in E^{2}
$$

and using the measurability of the function, we can split the integrals in equation (7.5) into $I_{1} \times I_{2}$, where:

$$
\begin{aligned}
& I_{1}=\int_{E} K_{1}\left(x_{1}, d x_{1}^{(1)}\right) \int_{E} \delta_{x_{1}^{(1)}}\left(d x_{2}^{(2)}\right) \\
& I_{2}=\int_{E^{2}} K_{2}\left(x_{2}, d x_{2}^{(1)}\right) \delta_{x_{2}^{(1)}}\left(d x_{1}^{(2)}\right) \min \left\{1, \frac{\pi_{1}\left(x_{2}^{(1)}\right)}{\pi_{2}\left(x_{2}^{(1)}\right) \varrho_{1}}\right\} K_{1}\left(x_{1}^{(2)}, A_{1}\right) .
\end{aligned}
$$

Clearly $I_{1}=1$. For $I_{2}$, integrating with respect to Dirac measure and then applying the minorization condition we obtain:

$$
I_{2} \geq \epsilon \int_{E} \nu\left(d x_{2}^{(1)}\right) \min \left\{1, \frac{\pi_{1}\left(x_{2}^{(1)}\right)}{\pi_{2}\left(x_{2}^{(1)}\right) \varrho_{1}}\right\} K_{1}\left(x_{2}^{(1)}, A_{1}\right) .
$$

Therefore equation (7.5) becomes:

$$
\widehat{K}\left(x_{1: 2}, A\right) \geq \theta \nu^{*}(A)
$$


where

$$
\begin{aligned}
\theta & =\epsilon^{2} \phi \\
\nu^{*}(A) & =K^{*}\left(A_{1}\right) \nu\left(A_{2}\right) \\
K^{*}\left(A_{1}\right) & =\frac{1}{\phi} \int_{E} \nu\left(d x_{2}^{(1)}\right) \min \left\{1, \frac{\pi_{1}\left(x_{2}^{(1)}\right)}{\pi_{2}\left(x_{2}^{(1)}\right) \varrho_{1}}\right\} K_{1}\left(x_{2}^{(1)}, A_{1}\right) \\
\phi & =\int_{E} \nu\left(d x_{2}\right) \min \left\{1, \frac{\pi_{1}\left(x_{2}\right)}{\pi_{2}\left(x_{2}\right) \varrho_{1}}\right\}
\end{aligned}
$$

Since (7.7) holds $\forall x_{1: 2} \in E^{2}, \forall A \in \mathcal{E} \otimes \mathcal{E}$ and the product measure is non-trivial, we have that $E^{2}$ is $\left(1, \theta, \nu^{*}\right)$ small, similarly $\widetilde{K}^{3}$ is uniformly ergodic.

Now suppose $\widehat{K}\left(x_{2: N}, d x_{2: N}\right)$ is uniformly ergodic (for arbitrary mixture parameters) under the specified conditions with $E^{N-1}((N-2), \epsilon, \nu)$ small. Let $\theta_{1}=\sum_{l=2}^{N} \varepsilon_{1 l}$. For $A=A_{1} \times \cdots \times A_{N}=A_{1: N} \in \mathcal{E}^{N}$, we have that:

$$
\begin{aligned}
\widehat{K}^{(N-1)}\left(x_{1: N}, A\right) \geq & \left(1-\theta_{1}\right)^{N-2} \int_{E^{N}} \widehat{K}\left(x_{1: N}, d x_{1: N}^{(1)}\right) \widehat{K}_{\theta_{1}}^{(N-2)}\left(x_{2: N}^{(1)}, A_{2: N}\right) \times \\
& K_{1}^{2 N-3}\left(x_{1}^{(1)}, A_{1}\right)
\end{aligned}
$$

where $\widehat{K}_{\theta_{1}}$ denotes that the mixture parameters in the exchange kernel have been modified by $\theta_{1}$. Equation (7.8) refers to the fact that the probability of moving to A under $\widehat{K}$ for any $x \in E^{N}$ is greater than considering the kernel that updates $x_{1}$ independently of $x_{2: N}$ (i.e. we can apply much the same arguments as for demonstrating $\left.\widetilde{K}^{3(N-1)}\left(x_{1: N}, A\right) \geq \tau^{2(N-1)}(1-\tau)^{N-1} \widehat{K}^{N-1}\left(x_{1: N}, A\right)\right)$. We then have:

$$
\widehat{K}^{(N-1)}\left(x_{1: N}, A\right) \geq \epsilon\left(1-\theta_{1}\right)^{N-2} \int_{E^{N}} \widehat{K}\left(x_{1: N}, d x_{1: N}^{(1)}\right) \nu\left(A_{2: N}\right) K_{1}^{2 N-3}\left(x_{1}^{(1)}, A_{1}\right) .
$$

At this point we may apply the above arguments to yield that $E^{N}$ is $\left(N-1, \theta, \nu^{*}\right)$ small for appropriate $\theta>0$ and probability measure $\nu^{*}$. Thus the result follows by induction and the fact that $\widetilde{K}^{3(N-1)}\left(x_{1: N}, A\right) \geq$ $\tau^{2(N-1)}(1-\tau)^{N-1} \widehat{K}^{N-1}\left(x_{1: N}, A\right)$.

Proof of Proposition Q Our approach is to focus upon the Dobrushin coeficient of $K_{M} \circ K_{E}$. Consider $N=2, f: E^{2} \rightarrow[0,1]:$

$$
\begin{gathered}
\left|K_{M} \circ K_{E}(f)\left(u_{1: 2}\right)-K_{M} \circ K_{E}(f)\left(v_{1: 2}\right)\right| \leq \mid \int_{E^{4}} \rho_{1,2}\left(x_{1}, x_{2}\right) f\left(x_{1: 2}^{\prime}\right) \delta_{x_{1}}\left(d x_{2}^{\prime}\right) \delta_{x_{2}}\left(d x_{1}^{\prime}\right) K_{M}\left(u_{1: 2}, d x_{1: 2}\right) \\
-\int_{E^{4}} \rho_{1,2}\left(x_{1}, x_{2}\right) f\left(y_{1: 2}^{\prime}\right) \delta_{x_{1}}\left(d y_{2}^{\prime}\right) \delta_{x_{2}}\left(d y_{1}^{\prime}\right) K_{M}\left(v_{1: 2}, d x_{1: 2}\right)|+| \int_{E^{4}}\left[1-\rho_{1,2}\left(x_{1}, x_{2}\right)\right] f\left(x_{1: 2}^{\prime}\right) \\
\delta_{x_{1}}\left(d x_{1}^{\prime}\right) \delta_{x_{2}}\left(d x_{2}^{\prime}\right) K_{M}\left(u_{1: 2}, d x_{1: 2}\right)-\int_{E^{4}}\left[1-\rho_{1,2}\left(x_{1}, x_{2}\right)\right] f\left(x_{1: 2}^{\prime}\right) \delta_{x_{1}}\left(d y_{1}^{\prime}\right) \delta_{x_{2}}\left(d y_{2}^{\prime}\right) K_{M}\left(v_{1: 2}, d x_{1: 2}\right) \mid \\
\leq\left[\operatorname{osc}\left(f \rho_{1,2}\right)+\operatorname{osc}\left(f\left[1-\rho_{1,2}\right]\right)\right] \beta\left(K_{M}\right) \\
\leq 2 \operatorname{osc}\left(\rho_{1,2}\right) \beta\left(K_{M}\right)
\end{gathered}
$$


where we have used the fact that $\operatorname{osc}(f g) \leq \sup (f) \operatorname{osc}(g)(\operatorname{since} \inf (f)=0$ and $\sup (f)=1)$. Note:

$$
\operatorname{osc}\left(\rho_{1,2}\right)=1-\inf _{\left(x_{1}, x_{2}\right) \in E^{2}} \rho_{1,2}\left(x_{1}, x_{2}\right)
$$

Now, since we have the mixing condition on $K_{M}$ we have that:

$$
\beta\left(K_{M} \circ K_{E}\right) \leq 2\left(1-\inf _{\left(x_{1}, x_{2}\right) \in E^{2}} \rho_{1,2}\left(x_{1}, x_{2}\right)\right)(1-\epsilon)
$$

Since

$$
\left\|\eta\left(K_{M} \circ K_{E}\right)^{n}-\pi^{*}\right\|_{T V} \leq \beta\left(\left(K_{M} \circ K_{E}\right)^{n}\right)\left\|\eta-\pi^{*}\right\|_{T V}
$$

(e.g. Del Moral (2004) chapter 4) we easily yield the desired result, for $N=2$. The result for $N \geq 3$ can be proved using the above arguments, with only notational complications.

\section{REFERENCES}

Andrieu, C. \& Moulines E. (2006). On the ergodicity properties of some adaptive MCMC algorithms. Ann. Appl. Prob.. 16, 1462-1505.

Bozdech, Z., Llinas, M., Pullium, B. L., Wong, E. D., Zhu, J. \& DeRisi, J. L. (2003). The transcriptome of the intraerythrocytic developmental cycle of Plasmodium falciparum. PLoS Biol. $1,1-16$.

Brooks, S. P., Giudici, P. \& Roberts, G. O. (2003). Efficient construction of reversible jump Markov chain Monte Carlo proposal distributions (with Discussion). J. R. Statist. Soc. B 65, 1-37.

Cappé, O., Robert, C. P. \& RydÉn, T. (2003). Reversible jump, birth-and-death and more general continuous time Markov chain Monte Carlo samplers. J. R. Statist. Soc. B 65, 679-700.

Carlin, B. \& Chib, S. (1995). Bayesian model choice via Markov chain Monte Carlo methods. J. $R$. Statist. Soc. B 57, 473-84.

Chauveau, D. \& Vandekerkhove, P. (2002). Improving convergence of the Hastings and Metropolis algorithm with an adaptive proposal. Scand. J. Statist., 29, 13-29.

Del Moral, P. (2004). Feynman-Kac Formulae: Genealogical and Interacting Particle Systems with Applications. Springer: New York.

Del Moral, P., Doucet, A., \& Jasra, A. (2006). Sequential Monte Carlo samplers. J. R. Statist. Soc. $B, \mathbf{6 8}, 411-36$. 
Dellaportas, P. \& Papageorgiou, I. (2006). Multivariate mixtures of normals with an unknown number of components. Statist. Comp., 16, 57-68.

Denison, D. G. T., Holmes, C. C., Mallick, B. K. \& Smith, A. F. M. (2002). Bayesian Methods for Nonlinear Classification and Regression. Chichester: Wiley.

Diaconis, P. \& Saloff-Coste, L. (1993). Comparison theorems for reversible Markov chains. Ann. Appl. Prob, 3, 696-750.

Dobrushin, R. L. (1956). Central limit theorem for non-stationary Markov chains I, II. Theory Probab. Appl., 1, 65-80, 329-83.

GEyer, C. (1991), Markov chain maximum likelihood, In Computing Science and Statistics: The 23rd symposium on the interface, (E. Keramigas ed), 156-63 Fairfax: Interface Foundation.

Geyer, C. \& Thompson, E. A. (1995). Annealing Markov chain Monte Carlo with applications to ancestral inference. J. Am. Statist. Assoc. 90, 909-20.

Gilks, W. R., Roberts, G. O. \& George, E. I. (1994). Adaptive direction sampling. The Statistician 43, 179-89.

Godsill, S. (2001). On the relationship between MCMC methods for uncertainty. J. Comput. Graph. Statist. 10, 230-48.

Green, P. J. (1995). Reversible jump Markov chain Monte Carlo computation and Bayesian model determination. Biometrika 82, 711-32.

Green, P. J. (2003a). Discussion of Efficient construction of reversible jump Markov chain Monte Carlo proposal distributions. J. R. Statist. Soc. B, 65, 48-9.

Green, P. J. (2003b). Trans-dimensional Markov chain Monte Carlo. In Highly Structured Stochastic Systems, (P.J. Green, N.L. Hjort \& S. Richardson eds), 179-96 Oxford: Oxford University Press.

Green, P. J. \& Mira, A. (2001). Delayed rejection in reversible jump Metropolis-Hastings. Biometrika $\mathbf{8 8}, 1035-53$.

Hastings, W. K. (1970). Monte Carlo sampling methods using Markov chains and their applications. Biometrika 57, 97-109.

Heard, N. A., Holmes, C. C., \& Stephens, D. A. (2006). A quantitative study of gene regulation involved in the immune response of anopheline mosquitoes: An application of Bayesian hierarchical clustering of curves. J. Amer. Statis. Assoc., 101, 18-29. 
Hodgson, M. E. A. (1999). A Bayesian restoration of an ion channel signal. J. R. Statist. Soc. B 61, $95-114$.

Hukushima, K. \& Nemoto, K. (1996). Exchange Monte Carlo method and application to spin glass simulations. J. Phys. Soc. Japan 65, 1604-08.

IbA, Y. (2001). Extended ensemble Monte Carlo. Int. J. Mod. Phys. 12, 653-56.

Jasra, A., Holmes, C. C., \& Stephens, D. A. (2005). Markov chain Monte Carlo methods and the label switching problem in Bayesian mixture modelling. Statist. Sci. 20, 50-67.

Jennison, C., Hurn, M. A. \& Al-Awadhi, F. (2003). Discussion of Efficient construction of reversible jump Markov chain Monte Carlo proposal distributions. J. R. Statist. Soc. B, 65, 44-5.

Kou, S. C., Zhou, Q. \& Wong, W. H. (2006). Equi-energy sampler with applications in statistical inference and statistical mechanics. Ann. Statist., 34, 1581-1619.

Liang, F. \& Wong, W. H. (2001). Real parameter evolutionary Monte Carlo with applications to Bayesian mixture models. J. Am. Statist. Assoc. 96, 653-66.

Liu, J. S. (2001). Monte Carlo Strategies in Scientific Computing. New York: Springer.

Madras, N. \& Zheng, Z. (2003). On the swapping algorithm. Random Structures and Algorithms 22, 66-97.

McLachlan, G. J. \& Peel, D. (2000). Finite Mixture Models. Chichester: Wiley.

Mengersen, K. L. \& Tweedie, R. L. (1996). Rates of convergence of the Hastings and Metropolis algorithms. Ann. Statist. 24, 101-21.

Metropolis, N., Rosenbluth, A. W., Rosenbluth, M. N., Teller, A. H. \& Teller, E. (1953). Equations of state calculations by fast computing machines. J. Chem. Phys. 21, 1087-92.

Richardson, S. \& Green, P. J. (1997). On Bayesian analysis of mixture models with an unknown number of components (with Discussion). J. R. Statist. Soc. B 59, 731-92.

Robert, C. P. \& Casella G. (2004). Monte Carlo Statistical Methods. Second edition. New York: Springer.

Roberts, G. O. \& Rosenthal, J. S. (2004). General state space Markov chains and MCMC algorithms. Prob. Surveys, 1, 20-71. 
Stephens, M. (2000). Bayesian analysis of mixture models with an unknown number of components - an alternative to reversible jump methods. Ann. Statist. 28, 40-74.

Tierney, L. (1994). Markov chains for exploring posterior distributions (with Discussion). Ann. Statist. 22, 1701-62.

Zheng, Z. (2003). On swapping and simulated tempering algorithms. Stoch. Proc. Appl. 104, 131-53. 